\title{
CORIOLIS MASS FLOW, DENSITY AND TEMPERATURE SENSING WITH A SINGLE VACCUM SEALED MEMS CHIP
}

\author{
D.Sparks, R.Smith, S.Massoud-Ansari, N.Najafi \\ Integrated Sensing Systems Inc., 391 Airport Industrial Dr, \\ Ypsilanti, MI 48198 www.mems-issys.com
}

\begin{abstract}
A new microfluidic system for measuring the mass flow rate, dose, chemical concentration, fluid density, specific gravity and temperature has been developed. Vacuum packaged, resonating silicon microtubes are employed to form both a Coriolis mass flow and density sensor. The micro Coriolis mass flow sensor has 10 times the resolution of the best commercially available macro Coriolis mass flow sensors. An onchip temperature sensor (RTD) has been added to the microchip, enabling accurate fluid temperature monitoring. At constant temperature the density resolution/or output stability approaches 1 over 1 million (6 digits) in the new system. Also a new method of chip-level gettering was developed to achieve the milliTorr pressures needed for adequate resonator signal quality for the vacuum packaged microsensors. Applications for both mass flow measurements and density sensing are discussed.
\end{abstract}

\section{INTRODUCTION}

Conventional Coriolis mass flow sensors have been commercially available for over 30 years. The basic function of an ideal resonating Coriolis mass flow sensor can be expressed by the following equations and is illustrated in Fig. 1. The mass flow rate $q$ is given by:

$q=K_{s} \vartheta /(4 \omega \mathbf{L r})$

Where, $\mathrm{K}_{\mathrm{s}}$ angular spring constant of the flow tube, $\vartheta$ is the twisting angle of the tube, $\omega$ is the resonance frequency, $\mathrm{L}$ is the length of the tube and $r$ is the radius of the U-bend of the tube. Therefore, the mass flow rate is directly proportional to the twisting angle and inversely proportional to the resonance frequency. The density of a liquid $\rho$ is given by the expression:

$\rho=1 / V\left[\left(K_{s} / 4 \pi^{2} f^{2}\right)-m_{t}\right]$

where $\mathrm{V}$ is the internal volume of the resonant tube, $\mathrm{m}_{\mathrm{t}}$ is tube mass, $K_{s}$ is the spring constant of the tube and $f$ is the resonance frequency of the tube. As can be seen by the expression above, the density is inversely proportional to the square of the resonance frequency.
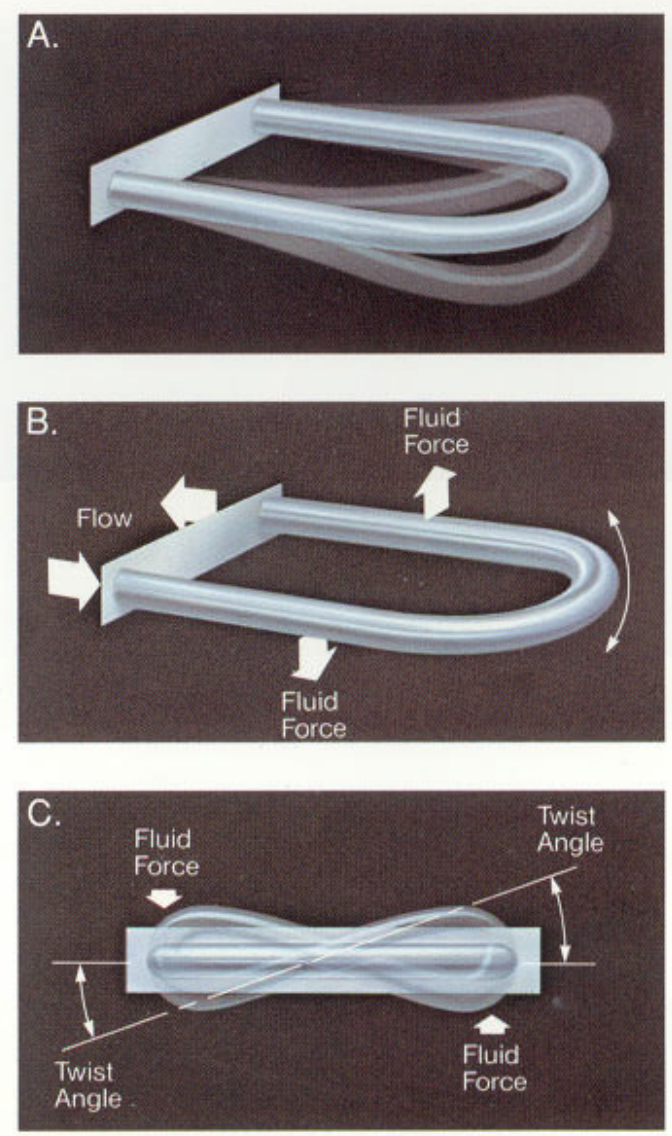

Fig. 1. Explanation of the Coriolis mass flow sensor tube motion: (a) vibration in the primary mode - used for density measurements, (b) forces acting on the sensing tube in upward motion, (c) end-on view of the sensor tube indicating force couple and tube twist.

The microfluidic sensor discussed in this paper is based on a MEMS chip concept first published in 2001 [1]. This early work as well as one other MEMS study [2] entailed the development of an R\&D micromachined chip only, and employed a laboratory vacuum chamber and instrumentation to both enable and sense resonance. At the core of the new sensor is a U-shaped, vacuum-sealed resonating silicon microtube. The fabrication process uses a combination of plasma and wet etching, photolithography, along with various types of waferto-wafer bonding to form the microfluidic chips [3-5]. While filled with a fluid, the tube is driven into 
resonance electrostatically and its motion sensed capacitively using metal electrodes under the tube and accompanying electronic circuits connected to the MEMS chip via wire bonding.

\section{MICROMACHINED SENSOR FABRICATION}

The silicon MEMS fabrication process used to form the microtubes has been described in previous publications $[3,4]$. A photograph of the resonant microtube chip is shown in Fig. 2. The doped silicon tube is anodically bonded to a metallized Pyrex wafer. Holes for the passage of fluid are predrilled into the Pyrex wafer. The metal pattern on the Pyrex forms capacitor plates, feedthroughs, electrical runners, a temperature sensor and silicon contacts.

A quality factor or Q value above 1000 was desired to obtain sufficient frequency and hence density resolution with the sensor. Initial test data on $R \& D$ samples were made using a vacuum chamber and

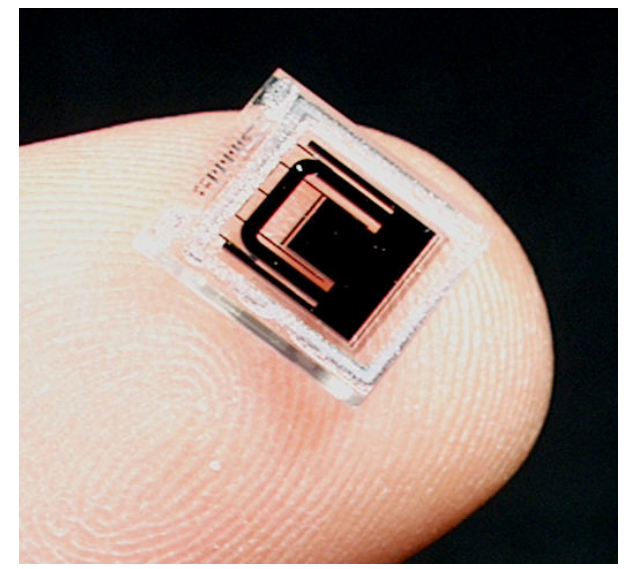

Fig. 2. The U-shaped microfluidic chip.

indicated that a cavity pressure of under $100 \mathrm{mTorr}$ would be needed to obtain an acceptable sensor signal. Conventional anodic bonding produces cavity pressures in the 100 - 400 Torr range [6,7], while glass frit and solder sealing produces cavity pressures of 1-2 Torr [8]. For this microfluidic device, a cavity pressure of 1.4 Torr was obtained with glass frit sealing and due to squeeze-film damping and molecular interaction, the Q value was limited to 40 for this wide vertical resonator.

After initially using NonEvaporable Getters (NEGs) with poor results, a new approach to MEMS gettering was developed [9-11]. This method was integrated into a wafer bonding process. A capping wafer, generally either silicon or glass was patterned and etched to form both a cavity that encloses the active micromachine and opens up access to the electrical bond pads. At this point the getter, called NanoGetters ${ }^{\mathrm{TM}}$, was applied and patterned on the top portion of the cavity, as shown in Fig. 3. The NanoGetter ${ }^{\mathrm{TM}}$ is comprised of a proprietary, patent

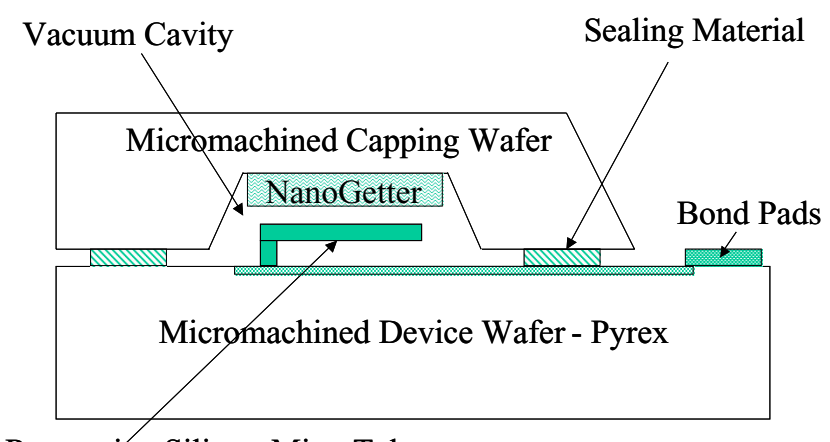

Resonating Silicon MicroTube

Fig. 3. Cross-sectional illustration of the vacuum packaging and gettering approach.

pending, multi-layer structure and as the name implies the thickness of the film layers are in the nanometer $5 \mathrm{~nm}$ to $500 \mathrm{~nm}$ range. Since thin film deposition techniques are employed in a cleanroom environment, the new getter is virtually particle free compared to conventional NEGs formed using powder metallurgy. The thin film deposition method also enhances the ability to easily integrate the getter into most typical MEMS processes.

\section{RESULTS}

Through wafer-to-wafer bonding with getters, the vacuum level obtained was found to be under a milliTorr, resulting in Q values ranging from 2000 to 45,000 for the silicon tube resonator. Extensive life testing of the hermetic glass frit seal and getter has been performed to insure that the basic MEMS device is reliable. Fig. 4 shows that the average $\mathrm{Q}$ of the resonators did not vary in a statistically significant manner whether the parts were at room temperature or

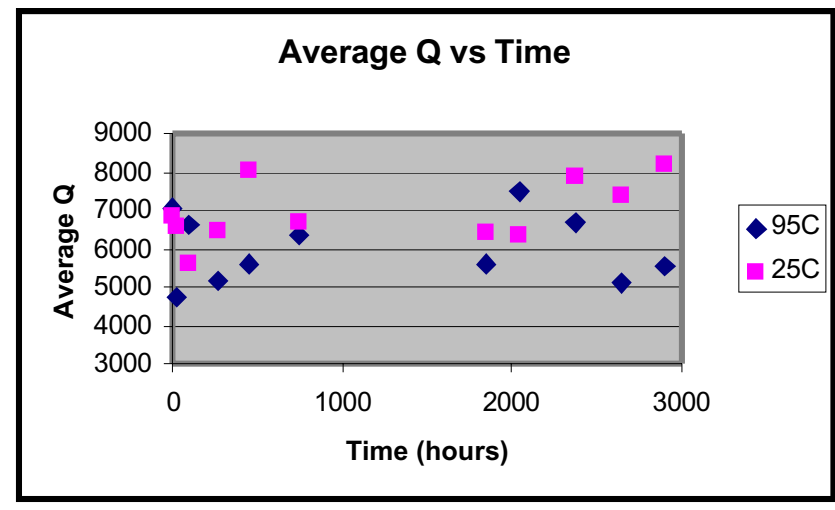

Fig. 4. Average $\mathrm{Q}$ versus time at $25^{\circ} \mathrm{C}$ and $95^{\circ} \mathrm{C}$ for 24 silicon resonators vacuum packaging using the NanoGetter ${ }^{\mathrm{TM}}$.

elevated temperature. In previous resonator reliability studies, Q values were found to decrease at high temperature when getters were not employed due to gas desorption from the microcavity walls [8]. 
The first product that was developed with this technology was a static density meter $[3,12]$. The commercially available density meter is shown in Fig 5. The development of a density meter was simpler and hence faster than that of a Coriolis mass flow sensor since the tube twisting did not have to be monitored. The resonant frequency of the microtube varies from $11 \mathrm{KHz}$ empty, down to $6 \mathrm{KHz}$ when filled with a dense liquid.

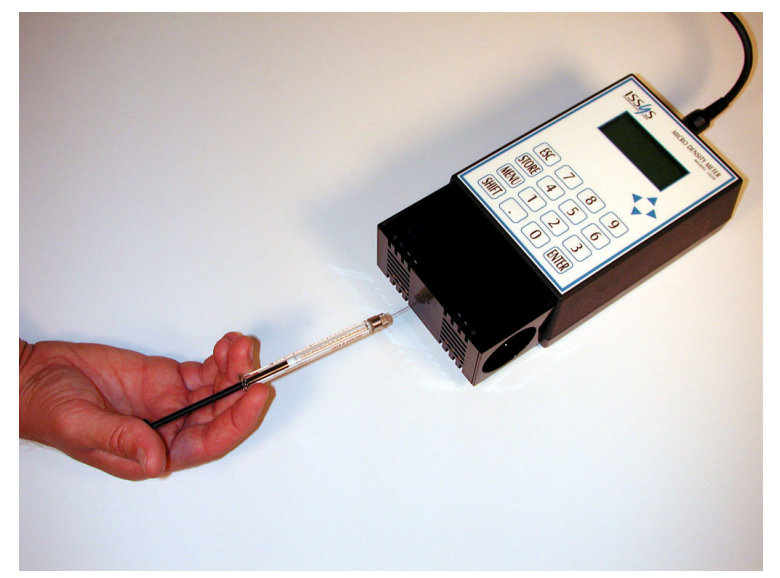

Fig. 5. Syringe loading of the temperature controlled microfluidic sensing system [3].

The system has been used to measure various petrochemicals, pharmaceuticals, beverages and aggressive chemical reagents. Micro density testing offers an advantage in saving time and money in distillation and fermentation applications as well as with the analysis of very expensive, small samples

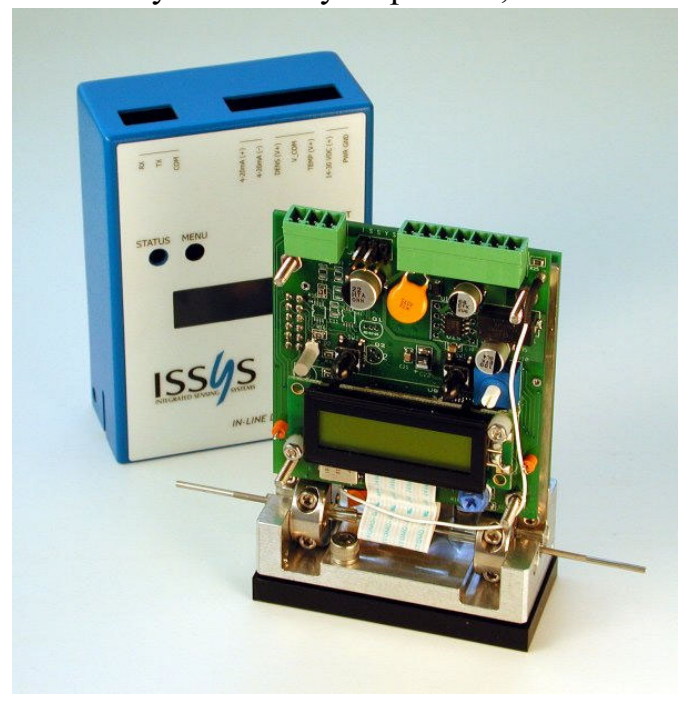

Fig. 6. Industrial in-line flow and density meter. such as perfumes and drug library samples.

Chemical concentration can be measured with this MEMS-based instrument using a mass-based solution approach. Sodium chloride (saltwater/saline) solutions are used for intravenous drug infusion.
Sucrose and dextrose solutions are employed in IV drug infusion as well as in beverage and food processing. Measurement of alcohol solutions see application in fuel cells, fuel blending and alcoholic beverages. An in-line meter has followed the launching of the static density meter. The same chassis used for this in-line density meter has been adapted for the Coriolis mass flow sensor. Fig. 6 shows the in-line package housing with the outer module casing removed.

Mass flow tests were performed using both nitrogen and argon gas. An ATC IL-05CC, traceable gas calibration sensor was used to measure the flow data. Fig. 7 shows the results of this initial testing. Good sensor output linearity was observed. Further improvement at the system board level is underway to

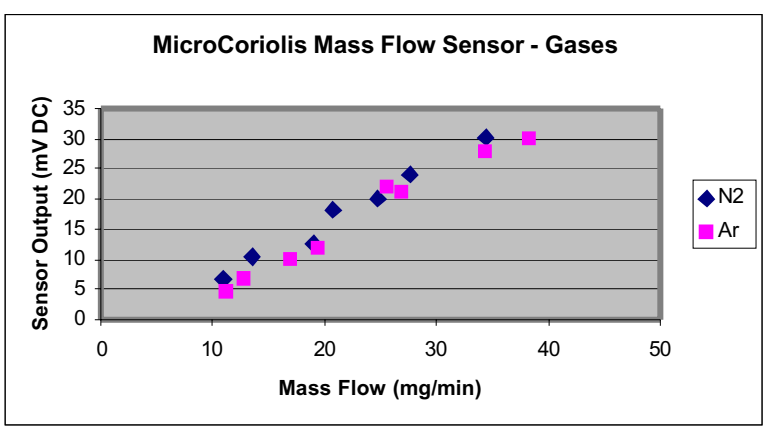

Fig. 7. Micro Coriolis mass flow output for gases.

reduce noise and improve overall sensor accuracy of these prototypes.

The same sensing elements have been used to measure the mass flow of water, methanol and various solutions. Fig. 8 shows how for liquid flow

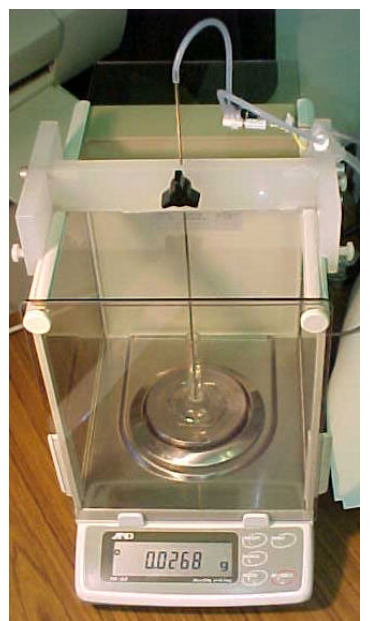

Fig. 8. Microscale system used to measure very low liquid mass flow rates.

monitoring a microscale was employed to measure the true mass of the liquid as it travels through the sensor and associated tubing. Precautions against air-flow related errors, temperature variation and evaporation were taken into account in this test system. The sensor was connected to a commercially available elastomeric drug infusion pump, rated at $0.5 \mathrm{~mL} / \mathrm{hr}$. These low 
pump supplied flow rates were measurable with this new sensor and confirmed with the microscale. This same microtube device has measured flow rates as in excess of $110 \mathrm{ml} / \mathrm{hr}$ using a gravity fed and syringe supplied fluid test system. In comparison the lowest flow rate, commercially available Coriolis mass flow sensor, the Quantim, which uses small metal tubes, has a minimum flow rate limit of $5 \mathrm{ml} / \mathrm{hr}$ [14]. Another MEMS Coriolis flow sensor developed by Enoksson et al. [2], in which the tubes were fabricated using wet silicon etching, had minimum flow rate measurements of $300 \mathrm{ml} / \mathrm{hr}$, due to the relatively large silicon tube size. Being able to measure flow rates below $100 \mathrm{ml} / \mathrm{hr}$, along with the sensor's small size and low power consumption, make this technology ideal for drug infusion applications. By integrating the mass flow with respect to time, the delivered dose and dose rate can be monitored. This sensor offers improved drug infusion safety features as well as a lower dose delivery capability.

One of the benefits of using Coriolis mass flow sensors is that a fluid density or specific gravity output is available. This can enable the user to monitor what type of fluid is traveling through the sensor. In medical applications this can prevent the delivery of the wrong drug and gas bubbles. Other applications for this low flow rate and density sensing technology are also found in the areas of drug discovery and development, leak detection, and the mixing and metering of industrial chemicals like pesticides, perfumes, flavor agents, fuels, dopants and additives.

\section{CONCLUSIONS}

A new microfluidic system for measuring the mass flow rate, dose, chemical concentration, fluid density, specific gravity and temperature has been developed. Vacuum packaged, resonating silicon microtubes are employed to form both a density meter and a Coriolis mass flow sensor. An on-chip temperature sensor has been added to the microchip, enabling accurate fluid temperature monitoring. As part of this development effort, a new method of gettering was developed to achieve the sub-milliTorr pressures needed for adequate resonator signal quality. With this NanoGetter ${ }^{\mathrm{TM}}$, Q values above 45,000 were obtained. MEMS-based, Coriolis mass flow and density sensors have been developed that are suitable for medical, industrial and laboratory applications.

\section{ACKNOWLEDGMENTS}

The authors would like to acknowledge the financial support of the Michigan Life Science Corridor, National Institute of Standards and Testing, and assistance in gas testing provided by ATC personnel.

\section{REFERENCES}

1. Y. Zhang, S. Tadigadapa and N. Najafi, "A micromachined Coriolis-force-based mass flowmeter for direct mass flow and fluid density measurements," Transducers'01, p.1460, 2001.

2. P. Enoksson, G. Stemme and E. Stemme, "A silicon resonant sensor structure for Coriolis mass-flow measurements, J.MEMS, vol. 6, p.119, 1997.

3. D. Sparks, R. Smith, M.Straayer, J.Cripe, R.Schneider, A.Chimbayo, S.Ansari, N.Najafi, "Measurement of density and chemical concentration using a microfluidic chip," Lab Chip, vol. 3, p.19, 2003.

4. S.Tadigadapa, C.Tsai, Y.Zhang and N.Najafi, "Micromachined fluidic apparatus," US Patent 6,477,901, 2002.

5. D.Sparks, "Integrated microtube sensing device," US Patent 6,647,778, 2003.

6. H.Henmi, S.Shoji, Y.Shoji, K.Yoshimi and M. Esashi, "Vacuum packaging for microsensors by glass-silicon anodic bonding," Sensors and Actuators A, vol. 43, p. 243, 1994.

7. M.Esashi, et al. "Vacuum-sealed silicon micromachined pressure sensors," Proc. IEEE, vol. 86, no. 8, p.1627, 1998.

8. D.Sparks, et al., "Wafer-to-wafer bonding of nonplanarized MEMS surfaces using solder," J. Micromech.\&Microengr., vol. 11, no. 6, p.630, 2001.

9. D. Sparks, S. Massoud-Ansari, N. Najafi, "Chiplevel vacuum packaging of micromachines using nanoGetters," IEEE Trans. Adv. Packaging, vol. 26, no. 3, p.277, Aug. 2003.

10. www.nanogetters.com

11. N.Najafi, S. Massoud-Ansari, S.Tadigadapa, and Y.Zhang, "Methods for prevention, reduction, and elimination of outgassing and trapped gases in micromachined devices," US Patent 6,499,354, 2002.

12. D.Sparks,R.Smith,M.Straayer,J.Cripe,R.Schneide, S.Ansari,N.Najafi, "A microfluidic system for the measurement of chemical concentration and density," Transducers'03, Boston, MA, 2C2.5, p. 300, 2003.

13. M.Tanaka et al., "Recommended table for the density of water between $0^{\circ} \mathrm{C}$ and $40^{\circ} \mathrm{C}$ based on recent experiemental reports," Metrologia, Vol. 38, p.301, 2001.

14. Quantim Coriolis Mass Flow Sensor, http://www.brooksinstrument.n1/PDS/QM/QmIP4 0-Pe-0102.pdf, Jan. 2002. 